\title{
UNIFORM ESTIMATION OF THE EIGENVALUES OF STURM-LIOUVILLE PROBLEMS
}

\author{
JOHN PAINE and FRANK DE HOOG
}

(Received 30 November 1978)

(Revised 1 May 1979)

\begin{abstract}
The perturbation of the eigenvalues of a regular Sturm-Liouville problem in normal form which results from a small perturbation of the coefficient function is known to be uniformly bounded. For numerical methods based on approximating the coefficients of the differential equation, this result is used to show that a better bound on the error is obtained when the problem is in normal form. A method having a uniform error bound is presented, and an extension of this method for general Sturm-Liouville problems is proposed and examined.
\end{abstract}

\section{Introduction}

The basic second-order Sturm-Liouville eigenvalue problem has the form

$$
\begin{gathered}
-\left(p u^{\prime}\right)^{\prime}+q u=\lambda r u, \quad x \in(a, b), \\
\alpha u(a)+\beta u^{\prime}(a)=0, \\
\gamma u(b)+\delta u^{\prime}(b)=0,
\end{gathered}
$$

$$
u, p u^{\prime} \in C[a, b]
$$

and this problem is said to be regular if $a$ and $b$ are finite, $\operatorname{pr} \in C^{2}[a, b], q \in C[a, b]$ and $p r>0$.

Problems of this form arise in many situations in mathematical physics and the information required about the spectrum varies with the application. The motivation for this paper lies in the need to determine the first $n(n \gg 1)$ free oscillation frequencies with a uniformly bounded error for various earth models (see, for example, [1], [18]). For such situations the use of standard techniques, such as finite difference and variational methods, does not guarantee the required type of approximation. 
The reasons for this are best illustrated by considering the use of these methods for solving

$$
\begin{gathered}
-u^{\prime \prime}=\lambda u, \quad x \in(0, \pi), \\
u(0)=0=u(\pi),
\end{gathered}
$$

which has eigenvalues $\lambda_{k}=k^{2}$ and eigenfunctions $u_{k}=\sin k x$. Then, on a uniform grid with step size $h=\pi / N$, we have the following error bounds for the following approximations $\tilde{\lambda}_{k}$ of $\lambda_{k}$.

\section{(A) Standard Central Differences}

It is well known [8] that

and hence

$$
\tilde{\lambda}_{k}=2 h^{-2}(1-\cos k h), \quad k=1,2, \ldots, N-1,
$$

$$
\left|\frac{\lambda_{k}-\tilde{\lambda}_{k}}{\lambda_{k}^{2}}\right| \leqslant \frac{h^{2}}{12}, \quad k=1,2, \ldots, N-1 .
$$

(B) Raleigh-Ritz Method with a Linear Hat Basis

In Schultz [16, p. 120] it is shown that

and hence

$$
\tilde{\lambda}_{k}=\frac{6(1-\cos k h)}{h^{2}(2+\cos k h)}, \quad k=1,2, \ldots, N-1,
$$

$$
\left|\frac{\tilde{\lambda}_{k}-\hat{\lambda}_{k}}{\dot{\lambda}_{k}^{2}}\right| \leqslant \frac{7 h^{2}}{12}, \quad k=1,2, \ldots, N-1 .
$$

(C) Rayleigh-Ritz Method with a Hermite Cubic Spline Basis

From [2, equation (31)] it follows that

$$
\left|\frac{\tilde{\lambda}_{k}-\lambda_{k}}{\sum_{j=1}^{k} \lambda_{j}^{4}}\right| \leqslant C h^{6}, \quad h<h_{0}(k)
$$

where (here and below) $C$ is a constant which is bounded independently of $k$ and $h$.

From these bounds it is clear that these methods will, with little effort, provide good approximations to the smaller eigenvalues. However, it is also clear that the amount of effort required to obtain uniform approximations of the first $n$ eigenvalues will be orders of magnitude larger than $n$. Thus, in the previously mentioned geophysical situation where fifty or one hundred eigenvalues are required, the use of standard methods will be inefficient because of the excessive computational effort required.

The deterioration in the accuracy of $\tilde{\lambda}_{k}$ with increasing $k$ in these cases is a direct consequence of the fact that the eigenfunctions become more oscillatory as the eigenvalue $\lambda_{k}$ increases. As each method relies on either a fixed-order polynomial or Taylor's series to approximate the eigenfunction, it is to be expected 
that the global accuracy of such approximations, and hence the eigenvalue approximation also will deteriorate as $k$ increases.

An alternative approach based on approximating the differential equation itself has been suggested by a number of authors (see, for example, [3], [7], [9], [13], [14], [15]). Specifically, we replace $p, q$ and $r$ in (1.1) by the approximations $\tilde{p}, \tilde{q}$ and $\tilde{r}$ respectively. Then we calculate the approximations to the eigenvalues and eigenfunctions by solving

$$
\begin{gathered}
-\left(\tilde{p} u^{\prime}\right)^{\prime}+\tilde{q} u=\lambda \tilde{r} u, \quad x \in(a, b), \\
\alpha u(a)+\beta u^{\prime}(a)=0, \\
\gamma u(b)+\delta u^{\prime}(b)=0,
\end{gathered}
$$

Let us neglect, for the time being, the difficulties associated with solving (1.2) and look at some of the features of such an approach.

Potentially, for a given approximation, we can obtain estimates of all the eigenvalues. It is not clear, however, what effect these perturbations of the coefficients will have on the eigenvalues, nor is it obvious that (1.2) will be any easier to solve than the original problem.

In an extensive analysis of such methods, Pruess [14] has shown that for piecewise $M$ th order polynomial interpolation of the coefficients on a grid with maximum stepsize $h$,

$$
\left|\lambda_{k}-\tilde{\lambda}_{k}\right| \leqslant C h^{M+1}\left|\lambda_{k}\right|, \quad h<h_{0} .
$$

He also extends this basic result to show that for piecewise polynomial interpolation at the Gaussian points

where

$$
\left|\lambda_{k}-\tilde{\lambda}_{k}\right| \leqslant C h^{2 M+2} \mid \lambda_{k}^{\alpha} \dot{j}, \quad h<h_{0}(k),
$$

$$
\alpha=\max \left\{1+\frac{1}{2} M, 3\right\} .
$$

For $M>0$, we can see that the above bounds represent a considerable improvement over the bounds obtained when finite difference and variational schemes are employed. However, in order to solve (1.2) it is necessary to obtain the fundamental solutions on each subinterval. If $M>0$ however, there does not in general exist a closed form for these fundamental solutions which is computationally convenient. Of course, a series solution could be developed, but this means that we are approximating the fundamental solutions by means of a piecewise polynomial and this is exactly the thing we are trying to avoid. It is only when $M=0$ that computationally tractable schemes can be constructed (see, for example, [3]). However, in this case we have

$$
\left|\lambda_{k}-\tilde{\lambda}_{k}\right| \leqslant C h\left|\lambda_{k}\right|
$$


for a general approximation, or if midpoint interpolation is used,

$$
\left|\lambda_{k}-\tilde{\lambda}_{k}\right| \leqslant C h^{2}\left|i_{k}^{3}\right|
$$

and so it is not clear that this scheme will be superior to conventional schemes.

In some special situations, the above bounds have been improved. For instance, when (1.1) is in Liouville normal form (that is, $p \equiv 1, r \equiv 1$ ) and $q$ is approximated on a unjform grid by midpoint interpolation, then Ixaru [10] has shown that

$$
\left|\frac{\lambda_{k}-\tilde{\lambda}_{k}}{\lambda_{k}^{\frac{1}{2}}}\right| \leqslant C h^{2}, \quad h<h_{0}
$$

Clearly, this is an improvement over the above estimates, but we still have not achieved the desired uniformity.

In this paper we use a standard perturbation argument to show that for problems in Liouville normal form, the approximations generated by (1.2) (with $\tilde{p} \equiv 1$, $\tilde{r} \equiv 1$ ) satisfy

$$
\left|\lambda_{k}-\tilde{\lambda}_{k}\right| \leqslant\|q-\tilde{q}\|_{2}, \quad k=1,2, \ldots,
$$

provided $\|q-\tilde{q}\|_{2}<\frac{1}{2} \min _{k}\left|\lambda_{k+1}-\lambda_{k}\right|$. It is then shown that this bound can be further improved for a certain class of midpoint piecewise constant approximations, and a scheme is proposed which will give uniform $O\left(h^{2}\right)$ approximations to all eigenvalues. Finally, we extend this method to include the uniform approximation of the eigenvalues of (1.1).

\section{Approximation of differential equations in normal form}

In this section we restrict attention to eigenvalue problems which are in Liouville normal form

$$
-u^{\prime \prime}+s u=\lambda u, \quad u \in D,
$$

where $s$ is a continuous function and

$D=\left\{u \in L^{2}([0,1]): u^{\prime}, u\right.$ are absolutely continuous, $u^{\prime \prime} \in L^{2}([0,1]), \alpha u(0)+$ $\left.\beta u^{\prime}(0)=0, \gamma u(1)+\delta u^{\prime}(1)=0\right\}$.

We further consider approximating problems of the form

$$
-u^{\prime \prime}+\tilde{s} u=\lambda u, \quad u \in D,
$$

where $\tilde{s}$ is a piecewise continuous function.

Let $\left\{\lambda_{k}\right\}_{k=0}^{\infty},\left\{\tilde{\lambda}_{k}\right\}_{k=0}^{\infty}$ be the eigenvalues of (2.1) and (2.2) respectively, arranged in ascending order, and $\left\{u_{k}\right\}_{k=0}^{\infty},\left\{\tilde{u}_{k}\right\}_{k=0}^{\infty}$ be the corresponding eigenfunctions, 
scaled so that

and

$$
\left\|u_{k}\right\|_{2}=\left\|\tilde{u}_{k}\right\|_{2}=1
$$

$$
\left(u_{k}, \tilde{u}_{k}\right) \geqslant 0 .
$$

Then we have the following results,

THEOREM 2.1. Let $\rho=\min _{k}\left|\lambda_{k+1}-\lambda_{k}\right|>0$ and $\tilde{s}$ be such that $\|s-\tilde{s}\|_{2}<\frac{1}{2} \rho$. Then,

(i) $\left|\lambda_{k}-\tilde{\lambda}_{k}\right| \leqslant\|s-\tilde{s}\|_{2}, k=0,1,2, \ldots$,

(ii) $\left\|u_{k}-\tilde{u}_{k}\right\|_{2} \leqslant \frac{4}{\rho}\|s-\tilde{s}\|_{2}, k=0,1,2, \ldots$.

Proof. (i) The first assertion follows from a result of Kato [11, p. 291] which states that if $T$ is a self-adjoint operator and $B$ a bounded symmetric operator both acting in a Hilbert space $H$, then $T+B$ is self-adjoint, and

$$
\operatorname{dist}[\Sigma(T), \Sigma(T+B)] \leqslant\|B\|,
$$

where $\Sigma(T), \Sigma(T+B)$ are the spectra of the operators $T$ and $T+B$ respectively, and

$$
\operatorname{dist}[X, Y]=\max \left\{\sup _{x \in X} \inf _{y \in Y}|x-y|, \sup _{y \in Y} \inf _{x \in X}|x-y|\right\}
$$

for any two sets $X$ and $Y$ contained in $\mathbf{C}$.

Now if we define the operators $L$ and $\tilde{L}$ by

$$
\begin{array}{ll}
L u=-u^{\prime \prime}+s u, & u \in D, \\
\tilde{L} u=-u^{\prime \prime}+\tilde{s} u, & u \in D,
\end{array}
$$

then it is clear that $\tilde{L}=L-B$ where $B$ is the bounded symmetric operator defined by

$$
B u=(s-\tilde{s}) u
$$

From [17, Theorem 10.18], $L$ is self-adjoint and since $D \subset L^{2}([0,1])$, the conditions of Kato's theorem are satisfied and hence,

$$
\operatorname{dist}[\Sigma(L), \Sigma(\tilde{L})] \leqslant\|B\|_{2} \text {. }
$$

The ordering of the spectra and the bound on $\|s-\tilde{s}\|_{2}$ then imply

(ii) Let

$$
\left|\lambda_{k}-\tilde{\lambda}_{k}\right| \leqslant\|s-\tilde{s}\|_{2}, \quad k=0,1,2, \ldots
$$

$$
\Gamma_{k}=\left\{\lambda \in C:\left|\lambda-\lambda_{k}\right|=\rho\right\}, \quad k=0,1,2, \ldots
$$

As $\lambda_{k}$ and $\tilde{\lambda}_{k}$ are the only eigenvalues of $L$ and $\tilde{L}$ respectively on the disc bounded by $\Gamma_{k}$, and both eigenvalues are interior points of $\Gamma_{k}$, the projection operators

$$
P_{k}=-\frac{1}{2 \pi i} \oint_{\Gamma_{k}}(L-i I)^{-1} d \hat{\lambda}
$$


and

$$
\tilde{P}_{k}=-\frac{1}{2 \pi i} \oint_{\Gamma_{k}}(\tilde{L}-\lambda I)^{-1} d \lambda
$$

are well defined and

for any $f \in L^{2}([0,1])$.

$$
\begin{aligned}
& P_{k} f=\left(u_{k}, f\right) u_{k}, \\
& \tilde{P}_{k} f=\left(\tilde{u}_{k}, f\right) \tilde{u}_{k}
\end{aligned}
$$

Furthermore, it is easily verified that for $\lambda \in \Gamma_{k}$,

$$
(\tilde{L}-\lambda I)^{-1}=(L-\lambda I)^{-1}+(\tilde{L}-\lambda I)^{-1} B(L-\lambda I)^{-1},
$$

and hence, from (2.6) with $f=u_{k}$ and (2.4),

where

$$
\begin{aligned}
\left(\tilde{u}_{k}, u_{k}\right) \tilde{u}_{k} & =\tilde{P}_{k} u_{k} \\
& =P_{k} u_{k}+R_{k} u_{k},
\end{aligned}
$$

$$
R_{k}=-\frac{1}{2 \pi i} \oint_{\Gamma_{k}}(\tilde{L}-\lambda I)^{-1} B(L-\lambda I)^{-1} d \lambda
$$

That is, using (2.5) in the above,

$$
\left(\tilde{u}_{k}, u_{k}\right) \tilde{u}_{k}=u_{k}+R_{k} u_{k}
$$

From Kato $\left[11\right.$, p. 291] and the bound on $\|s-\tilde{s}\|_{2}$, we find that

and, from (2.7)

$$
\left\|R_{k}\right\|_{2} \leqslant \frac{2}{\rho}\|s-\tilde{s}\|_{2}<1
$$

But, also from (2.7),

$$
\left\|R_{k}\right\|_{2} \geqslant 1-\left(\tilde{u}_{k}, u_{k}\right) \geqslant 0 \text {. }
$$

and so

$$
\tilde{u}_{k}-u_{k}=\left(1-\left(\tilde{u}_{k}, u_{k}\right)\right) \tilde{u}_{k}+R_{k} u_{k}
$$

$$
\begin{aligned}
\left\|\tilde{u}_{k}-u_{k}\right\|_{2} & \leqslant 2\left\|R_{k}\right\|_{2} \\
& \leqslant \frac{4}{\rho}\|s-\tilde{s}\|_{2} .
\end{aligned}
$$

From these basic results it is also possible to derive a refined estimate of the eigenvalue perturbation which will be useful for obtaining higher order convergence results in the next section. This result is

THEOREM 2.2. If $\|s-\bar{s}\|_{2}<\frac{1}{2} \rho$ where $\rho$ is as defined in Theorem 2.1, then

$$
\left|\hat{\lambda}_{k}-\tilde{\lambda}_{k}-\int_{0}^{1}(s-\tilde{s}) u_{k}^{2} d x\right| \leqslant \frac{4}{\rho}\|s-\tilde{s}\|_{2}^{2}, \quad k=0,1,2, \ldots
$$


Proof. Let $k$ be a given integer. From (2.7) we have, since $\left\|R_{k}\right\|_{2}<1$ implies that $\left(\tilde{u}_{k}, u_{k}\right) \neq 0$,

$$
\tilde{u}_{k}=\left(u_{k}+R_{k} u_{k}\right) /\left(\tilde{u}_{k}, u_{k}\right)
$$

where $u_{k}$ and $\tilde{u}_{k}$ are the eigenfunctions of (2.1) and (2.2) respectively, corresponding to the eigenvalues $\lambda_{k}$ and $\tilde{\lambda}_{k}$.

Applying $L$ to $\tilde{u}_{k}$ and using (2.2) and (2.9) yields

$$
\tilde{L} \tilde{u}_{k}=\tilde{\lambda}_{k}\left(u_{k}+R_{k} u_{k}\right) /\left(\tilde{u}_{k}, u_{k}\right) \text {; }
$$

but, since $\tilde{L}=L-B$, we also have

Hence

$$
\tilde{L} \tilde{u}_{k}=\left(\lambda_{k} u_{k}-B u_{k}+\tilde{L} R_{k} u_{k}\right) /\left(\tilde{u}_{k}, u_{k}\right)
$$

$$
\left(\lambda_{k}-\tilde{\lambda}_{k}\right) u_{k}-B u_{k}=-\left(\tilde{L}-\tilde{\lambda}_{k} I\right) R_{k} u_{k}
$$

and on taking the inner product of both sides with $u_{k}$ and noting that $\left(u_{k}, u_{k}\right)=1$,

$$
\left(\hat{\lambda}_{k}-\tilde{\lambda}_{k}\right)-\int_{0}^{1}(s-\tilde{s}) u_{k}^{2} d x=-\left(u_{k},\left(\tilde{L}-\tilde{\lambda}_{k} I\right) R_{k} u_{k}\right)
$$

But, since $\left(\tilde{L}-\tilde{\lambda}_{k} I\right)$ is self-adjoint,

$$
\begin{aligned}
\left|\left(u_{k},\left(\tilde{L}-\tilde{\lambda}_{k} I\right) R_{k} u_{k}\right)\right| & =\left|\left(\lambda_{k}-\tilde{\lambda}_{k}\right)\left(u_{k}, R_{k} u_{k}\right)+\left(B u_{k}, R_{k} u_{k}\right)\right| \\
& \leqslant\left|\lambda_{k}-\tilde{\lambda}_{k}\right|\left\|R_{k}\right\|_{2}+\|B\|_{2}\left\|R_{k}\right\|_{2} .
\end{aligned}
$$

Theorem 2.1 and the inequality (2.8) then yield the desired result.

\section{Convergence results for problems in normal form}

From the preceding results, it is a simple matter to obtain results analogous to those of Pruess [14] when $s$ is approximated by a piecewise polynomial. However, due to the inherent difficulties in solving the approximate problem for the general case, we shall restrict attention to the case of piecewise constant approximation.

Let

$$
\Delta=\left\{x_{i}, i=0,1, \ldots N \mid x_{0}=0, x_{N}=1, x_{i+1}>x_{i}\right\}
$$

be a partition of $[0,1]$,

$$
h=\max _{0 \leqslant i \leqslant N-1}\left|x_{i+1}-x_{i}\right|,
$$

then the piecewise constant approximation $\tilde{s}$ is defined by

$$
\tilde{s}(x)=s_{i}, \quad x \in\left(x_{i}, x_{i+1}\right), \quad i=0,1, \ldots, N-1,
$$

where $s_{i}$ is some constant.

Then, from Theorem 2.1, 
Corollary 3.1. Let $s \in C^{1}[0,1], \Delta$ be a partition of $[0,1]$ and $s_{i}$ interpolate $s$ at some point of $\left[x_{i}, x_{i+1}\right]$ for each $i$. Then there is an $h_{0}>0$ such that for each partition $\Delta$ with $h<h_{0}$

$$
\left|\hat{\lambda}_{k}-\tilde{\lambda}_{k}\right| \leqslant\left\|s^{\prime}\right\|_{\infty} h, \quad k=0,1,2, \ldots
$$

Proof. If we define $h_{0}=\frac{1}{2} \rho /\left\|s^{\prime}\right\|_{\infty}$, then the result follows from Theorem 2.1 since $\|s-\tilde{s}\|_{\infty} \leqslant h\left\|s^{\prime}\right\|_{\alpha}$.

Similarly, if $s \in C^{2}[0,1]$ and $\tilde{s}$ is given by midpoint interpolation, then Ixauru's [10] result follows from Theorem 2.2, by using any standard result on the error of midpoint product integration. However, initial computational results indicated that for midpoint interpolation on a uniform grid, this bound can be improved. Before giving this improved bound, the following definitions and lemmas are required:

LEMMA 3.1. Let $\left\{\eta_{k}\right\}_{k=0}^{\infty}$ and $\left\{w_{k}\right\}_{k=0}^{\infty}$ be the ordered eigenvalues and eigenfunctions of the operator $L_{0}$, where $L_{0}$ is defined by

$$
L_{0} w=-w^{\prime \prime}, \quad w \in D,
$$

and where the eigenfunctions are scaled so that

and

$$
\left\|w_{k}\right\|_{2}=1
$$

$$
\left(w_{k}, u_{k}\right) \geqslant 0 \text {. }
$$

Then, there is a positive constant $C_{1}$, bounded independently of $k$, such that for each integer $k$,

(i) $\left\|u_{k}-w_{k}\right\|_{\infty} \leqslant C_{1} /(k+1)$,

(ii) $\left\|u_{k}^{\prime}-w_{k}^{\prime}\right\|_{\infty} \leqslant C_{1}$,

(iii) $\left\|w_{k}\right\|_{\infty} \leqslant C_{1}$,

(iv) $\left\|w_{k}^{\prime}\right\|_{\infty} \leqslant(k+1) C_{1}$.

Proof. All four inequalities follow directly from the asymptotic representation of the normalized eigenfunctions and their derivatives given in Courant and Hilbert [6, p. 336].

On a uniform partition $\Delta$ of $[0,1]$, define $s^{*}$ to be the piecewise constant approximation determined by the rule

where

$$
s^{*}(x)=s\left(x_{i+\frac{1}{2}}\right), \quad x \in\left(x_{i}, x_{i+1}\right),
$$

Then we have

$$
x_{i+\frac{1}{2}}=x_{i}+\frac{1}{2} h, \quad i=0,1, \ldots, N-1 \text {. }
$$


LeMma 3.2. Let $s \in C^{2}[0,1], f \in C^{1}[0,1]$ and $\Delta$ be a uniform partition of $[0,1]$, then

$$
\left|\int_{0}^{1}\left(s-s^{*}\right) f d x\right| \leqslant\left\{\frac{1}{4}\left\|s^{\prime} i_{\infty}\right\| f^{\prime}\left\|_{\infty}+\frac{1}{8}\right\| f^{\prime \prime}\left\|_{\infty}\right\| f \|_{\infty}\right\} h^{2} .
$$

Proof.

$$
\begin{aligned}
\left|\int_{0}^{1}\left(s-s^{*}\right) f d x\right| \leqslant & \sum_{i=0}^{N-1}\left|\int_{x_{i}}^{x_{i+1}}\left[s(x)-s\left(x_{i+\frac{1}{2}}\right)\right] f(x) d x\right| \\
\leqslant & \sum_{i=0}^{N-1}\left|\int_{x_{i}}^{x_{i+1}} s^{\prime}\left(x_{i+\frac{1}{2}}\right)\left(x-x_{i+\frac{1}{2}}\right) f\left(x_{i+\frac{1}{2}}\right) d x\right| \\
& +\sum_{i=0}^{N-1}\left|\int_{x_{1}}^{x_{i+1}} s^{\prime}\left(x_{i+\frac{1}{2}}\right)\left(x-x_{i+\frac{1}{2}}\right)\left[f(x)-f\left(x_{i+\frac{1}{2}}\right)\right] d x\right| \\
& +\sum_{i=0}^{N-1}\left|\int_{x_{i}}^{x_{i+1}}\left[s(x)-s\left(x_{i+\frac{1}{2}}\right)-s^{\prime}\left(x_{i+\frac{1}{2}}\right)\left(x-x_{i+\frac{1}{2}}\right)\right] f(x) d x\right| .
\end{aligned}
$$

Using the inequalities

and

$$
\left|s(x)-s\left(x_{i+\frac{1}{2}}\right)-s^{\prime}\left(x_{i+\frac{1}{2}}\right)\left(x-x_{i+\frac{1}{i}}\right)\right| \leqslant \frac{1}{8}\left\|s^{\prime \prime}\right\|_{\infty} h^{2}
$$

it is easy to verify that

$$
\left|f(x)-f\left(x_{i+\frac{1}{2}}\right)\right| \leqslant \frac{1}{2}\left\|f^{\prime}\right\|_{\infty} h,
$$

$$
\left|\int_{0}^{1}(s-\tilde{s}) f d x\right| \leqslant\left\{\frac{1}{4}\left\|s^{\prime}\right\|_{\infty}\left\|f^{\prime}\right\|_{\infty}+\frac{1}{8}\left\|f^{\prime \prime}\right\|_{\infty}\|f\|_{\infty}\right\} h^{2} .
$$

If we now define $\left\{\lambda_{k}^{*}\right\}$ and $\left\{u_{k}^{*}\right\}$ to be the eigenvalues and eigenfunctions of (2.2) with $\tilde{s}=s^{*}$, then we have

THEOREM 3.1. Let $s \in C^{2}[0,1]$, then there is an $h_{0}>0$ such that for each uniform partition $\Delta$ with $h<h_{0}$,

$$
\begin{array}{r}
\left|\lambda_{k}-\lambda_{k}^{*}\right| \leqslant C_{1}\left|\frac{\cos \sqrt{ }\left(\eta_{k}\right) h}{\sqrt{ }\left(\eta_{k}\right) h}-\frac{\sin \sqrt{ }\left(\eta_{k}\right) h}{\eta_{k} h^{2}}\right| \max _{1 \leqslant p \leqslant N-1}\left|\frac{\sin p \sqrt{ }\left(\eta_{k}\right) h}{\sin \sqrt{ }\left(\eta_{k}\right) h}\right| h^{2}+C_{2} h^{2}, \\
k=0,1,2, \ldots,
\end{array}
$$

where $C_{1}$ and $C_{2}$ are constants bounded independently of $k$ and $h$.

Proof. Let $h_{0}=\rho /\left\|s^{\prime}\right\|_{\infty_{-}}$and $\Delta$ be a uniform partition with $h<h_{0}$, then the conditions of Theorem 2.2 are satisfied since

Using the identity

$$
\left\|s-s^{*}\right\|_{\infty} \leqslant \frac{1}{2} h\left\|s^{\prime}\right\|_{\infty}<\frac{1}{2} \rho .
$$

$$
u_{k}^{2}=w_{k}^{2}+2\left(u_{k}-w_{k}\right) w_{k}+\left(u_{k}-w_{k}\right)^{2}
$$


in Theorem 2.2, we find

$$
\begin{aligned}
\left|\lambda_{k}-\lambda_{k}^{*}\right| \leqslant\left|\int_{0}^{1}\left(s-s^{*}\right) w_{k}^{2} d x\right|+2\left|\int_{0}^{1}\left(s-s^{*}\right)\left(u_{k}-w_{k}\right) w_{k} d x\right| \\
+\left|\int_{0}^{1}\left(s-s^{*}\right)\left(u_{k}-w_{k}\right)^{2} d x\right|+\frac{4}{\rho}\left\|s^{\prime}\right\|_{\infty}^{2} h^{2} .
\end{aligned}
$$

Estimating the last two integrals through the application of Lemma 3.2 together with the bounds of Lemma 3.1, then yields

$$
\left|\lambda_{k}-\lambda_{k}^{*}\right| \leqslant\left|\int_{0}^{1}\left(s-s^{*}\right) w_{k}^{2} d x\right|+C h^{2} .
$$

If on each of the intervals $\left(x_{i}, x_{i+1}\right)$ we replace $\left(s-s^{*}\right)$ by a Taylor's series expanded about $x_{i+\frac{1}{2}}$, we obtain

$$
\left|\int_{0}^{1}\left(s-s^{*}\right) w_{k}^{2} d x\right| \leqslant\left|\sum_{i=0}^{N-1} s^{\prime}\left(x_{i+\frac{1}{2}}\right) \int_{x_{i}}^{x_{i+1}}\left(x-x_{i+\frac{1}{2}}\right) w_{k}^{2} d x\right|+\frac{1}{8} h^{2}\left\|s^{\prime \prime}\right\|_{\infty} \int_{0}^{1} w_{k}^{2} d x
$$

and, since $w_{k}=A_{k} \sin \left(\sqrt{ }\left(\eta_{k}\right) x+\phi_{k}\right)$, we can integrate the above to give

$$
\begin{aligned}
\left|\int_{0}^{1}\left(s-s^{*}\right) w_{k}^{2} d x\right| \leqslant & \frac{A_{k}^{2} h^{2}}{4}\left|\frac{\cos \sqrt{ }\left(\eta_{k}\right) h}{\sqrt{ }\left(\eta_{k}\right) h}-\frac{\sin \sqrt{ }\left(\eta_{k}\right) h}{\eta_{k} h^{2}}\right| \\
& \times\left|\sum_{i=0}^{N-1} s^{\prime}\left(x_{i+\frac{1}{2}}\right) \sin \left(2 \sqrt{ }\left(\eta_{k}\right) x_{i+\frac{1}{2}}+2 \phi_{k}\right)\right|+\frac{1}{8} h^{2}\left\|s^{\prime \prime}\right\|_{\infty} .
\end{aligned}
$$

Now, on summing by parts,

$$
\begin{array}{r}
\sum_{i=0}^{N-1} s^{\prime}\left(x_{i+\frac{1}{2}}\right) \sin \left(2 \sqrt{ }\left(\eta_{k}\right) x_{i+\frac{1}{2}}+2 \phi_{k}\right)=s^{\prime}\left(x_{N-\frac{1}{2}}\right) \sum_{j=0}^{N-1} \sin \left(2 \sqrt{ }\left(\eta_{k}\right) x_{j+\frac{1}{2}}+2 \phi_{k}\right) \\
-\sum_{i=1}^{N-1}\left\{s^{\prime}\left(x_{i+\frac{1}{2}}\right)-s^{\prime}\left(x_{i-\frac{1}{2}}\right)\right\} \sum_{j=0}^{i-1} \sin \left(2 \sqrt{ }\left(\eta_{k}\right) x_{j+\frac{1}{2}}+2 \phi_{k}\right) .
\end{array}
$$

But since

$$
\left|\sum_{j=0}^{i-1} \sin \left(2 \sqrt{ }\left(\eta_{k}\right) x_{j+\frac{1}{2}}+2 \phi_{k}\right)\right| \leqslant 2\left|\frac{\sin i \sqrt{ }\left(\eta_{k}\right) h}{\sin \sqrt{ }\left(\eta_{k}\right) h}\right|
$$

and

$$
\left|s^{\prime}\left(x_{i+\frac{1}{2}}\right)-s^{\prime}\left(x_{i-\frac{1}{2}}\right)\right| \leqslant h\left\|s^{\prime \prime}\right\|_{\infty},
$$

(3.2), (3.3) and (3.4) give the desired result.

The presence of the term

$$
\max _{1 \leqslant p \leqslant N-1}\left|\frac{\sin p \sqrt{ }\left(\eta_{k}\right) h}{\sin \sqrt{ }\left(\eta_{k}\right) h}\right|
$$


in the above error bound makes it immediately obvious that this bound cannot be uniformly $O\left(h^{2}\right)$. In spite of this negative result, we will now show that by using the above approach to find the first [N/2] eigenvalues and then switching over to a simple asymptotic expansion, uniform $O\left(h^{2}\right)$ estimates of all eigenvalues can be found.

In [12] it is shown that

$$
\lambda_{k}=\eta_{k}+\int_{0}^{1} s d x+O\left(k^{-2}\right)
$$

and so it is clear that this expansion will provide uniform $O\left(h^{2}\right)$ approximations for $k=[N / 2],[N / 2]+1, \ldots$. Thus, we only need to show that the bound in Theorem (3.1) is uniform for $k=0,1, \ldots,[N / 2]$. But, from [6], $\sqrt{ } \eta_{k}=k \pi+O(1)$, thus

$$
\sqrt{ }\left(\eta_{k}\right) h<\frac{1}{2} \pi+O(h)
$$

and so

$$
\left|\frac{\cos \sqrt{ }\left(\eta_{k}\right) h}{\sqrt{ }\left(\eta_{k}\right) h}-\frac{\sin \sqrt{ }\left(\eta_{k}\right) \dot{h}}{\eta_{k} h^{2}}\right| \leqslant \sqrt{ }\left(\eta_{k}\right) h
$$

and

Therefore,

$$
\max _{1 \leqslant p \leqslant N-1}\left|\frac{\sin p \sqrt{ }\left(\eta_{k}\right) h}{\sin \sqrt{ }\left(\eta_{k}\right) h}\right| \leqslant \frac{\pi}{2 \sqrt{ }\left(\eta_{k}\right) h}+O(h)
$$

$$
\left|\dot{\lambda}_{k}-\lambda_{k}^{*}\right| \leqslant\left(\frac{1}{2} \pi C_{1}+C_{2}\right) h^{2}+O\left(h^{3}\right), \quad k=0,1, \ldots,[N / 2] .
$$

Consequently, the above approach does in fact yield uniform approximations to all eigenvalues.

\section{Numerical schemes for piecewise constant approximation}

When $\tilde{s}$ is chosen as a piecewise constant function, the solution of (2.2) for a given value of $\tilde{\lambda}$ is

$$
v_{\lambda}(x)=\left\{\begin{array}{ccc}
A_{0} F_{0}(x, \lambda)+B_{0} G_{0}(x, \lambda), & x \in\left[x_{0}, x_{1}\right] \\
\cdot & \cdot & \cdot \\
\cdot & \cdot & \cdot \\
\cdot & \cdot & \cdot \\
A_{N-1} F_{N-1}(x, \lambda)+B_{N-1} G_{N-1}(x, \lambda), & x \in\left[x_{N-1}, x_{N}\right]
\end{array}\right.
$$

where $F_{i}(x, \lambda)$ and $G_{i}(x, \lambda)$ are the fundamental solutions of

$$
-v^{\prime \prime}+s_{i} v=\lambda v, \quad x \in\left(x_{i}, x_{i+1}\right)
$$


which were chosen as

$$
\begin{gathered}
F_{i}(x, \lambda)= \begin{cases}\cos \left(\sqrt{ }\left(\lambda-s_{i}\right)\left(x-x_{i}\right)\right), & \lambda>s_{i}, \\
1, & i=s_{i}, \\
\cosh \left(\sqrt{ }\left(s_{i}-\lambda\right)\left(x-x_{i}\right)\right), & i<s_{i},\end{cases} \\
G_{i}(x, \lambda)= \begin{cases}\sin \left(\sqrt{ }\left(\lambda-s_{i}\right)\left(x-x_{i}\right)\right) / \sqrt{ }\left(i-s_{i}\right), & i>s_{i}, \\
\left(x-x_{i}\right), & i=s_{i}, \\
\sinh \left(\sqrt{ }\left(s_{i}-\lambda\right)\left(x-x_{i}\right)\right) / \sqrt{ }\left(s_{i}-\lambda\right), & \lambda<s_{i} .\end{cases}
\end{gathered}
$$

The constants $\left\{A_{i}, B_{i}\right\}_{i=0}^{N-1}$ are determined up to an arbitrary multiple by the additional requirement that $v_{\lambda} \in D$.

The determination of the eigenvalues of (2.2) thus becomes that of finding the values of $\lambda$ for which $\left\{A_{i}\right\}_{i=0}^{N-1}$ and $\left\{B_{i}\right\}_{i=0}^{N-1}$ are not identically zero. To do this, we note that the requirement $v_{\lambda} \in D$ yields a system of equations involving the unknowns $A_{i}, B_{i}, v_{\lambda}\left(x_{1}\right), v_{\lambda}{ }^{\prime}\left(x_{i}\right)$ and the known values $F_{i}\left(x_{i+1}, \lambda\right)$, and $G_{i}\left(x_{i+1}, \lambda\right)$. We can then proceed in a number of ways, for example, Canosa [3] uses these relations to eliminate the unknowns $\left\{v_{\lambda}\left(x_{i}\right), v_{\lambda}^{\prime}\left(x_{i}\right)\right\}_{i=0}^{N-1}$ and obtains a system of equations in $\left\{A_{i}, B_{i}\right\}_{i=0}^{N-1}$. The eigenvalues are then simply the zeros of the determinant of this system. However, if these relations are used to eliminate the unknowns $\left\{A_{i}, B_{i}\right\}_{i=0}^{N-1}$ and $\left\{v_{\lambda}^{\prime}\left(x_{i}\right)\right\}_{i=0}^{N-1}$ from the continuity conditions, we obtain $G_{i+1}\left(x_{i+2}, \lambda\right) v_{\lambda}\left(x_{i}\right)-\left[F_{i}\left(x_{i+1}, \lambda\right) G_{i+1}\left(x_{i+2}, \lambda\right)+F_{i+1}\left(x_{i+2}, \lambda\right) G_{i}\left(x_{i+1}, \lambda\right)\right] v_{\lambda}\left(x_{i+1}\right)$
$+G_{i}\left(x_{i+1}, \lambda\right) v_{\lambda}\left(x_{i+2}\right)=0, \quad i=0,1, \ldots, N-2$.

These relations, together with the equations

and

$$
\left[\beta F_{0}\left(x_{1}, \lambda\right)-\alpha G_{0}\left(x_{1}, \lambda\right)\right] v_{\lambda}\left(x_{0}\right)-\beta v_{\lambda}\left(x_{1}\right)=0
$$

$$
-\delta v_{\lambda}\left(x_{N-1}\right)+\left[\gamma G_{N-1}\left(x_{N}, \lambda\right)+\delta F_{N-1}\left(x_{N}, \lambda\right)\right] \bar{v}_{\lambda}\left(x_{N}\right)=0
$$

which are obtained in a similar fashion from the boundary conditions, yield a system of equations of the form

where

$$
D(\lambda) \mathbf{v}=\mathbf{0},
$$

$$
\mathbf{v}^{\mathbf{\top}}=\left(v_{\lambda}\left(x_{0}\right), v_{\lambda}\left(x_{1}\right), \ldots, v_{\lambda}\left(x_{N}\right)\right)
$$

and $D(\lambda)$ is a tridiagonal matrix.

This approach provides a simpler procedure for finding the eigenvalues of (2.2) and also yields a simple direct method for evaluating the eigenfunction at the knots of the partition. Unfortunately, there is the difficulty that not all zeros of the determinant are necessarily eigenvalues. To illustrate this, consider the problem

$$
\begin{gathered}
-v^{\prime \prime}=\lambda v, \\
v^{\prime}(0)=0=v(\pi),
\end{gathered}
$$

the exact eigenvalues of which are $\lambda_{k}=\left(k+\frac{1}{2}\right)^{2}, k=0,1,2, \ldots$. 
Now for the partition $\left\{0, \frac{1}{2} \pi, \pi\right\}$

$$
D(\lambda)=\left[\begin{array}{ccc}
\cos \frac{\sqrt{ }(\lambda) \pi}{2} & -1 & 0 \\
\frac{1}{\sqrt{ } \lambda} \sin \frac{\sqrt{ }(\lambda) \pi}{2} & -\frac{2}{\sqrt{ } \lambda} \sin \frac{\sqrt{ }(\lambda) \pi}{2} \cos \frac{\sqrt{ }(\lambda) \pi}{2} & \frac{1}{\sqrt{ } \lambda} \sin \frac{\sqrt{ }(\lambda) \pi}{2} \\
0 & 0 & 1
\end{array}\right]
$$

and so,

$$
\operatorname{det}(D(i))=-\frac{1}{\sqrt{ } \lambda} \sin \frac{\sqrt{ }(\lambda) \pi}{2} \cos \sqrt{ }(\lambda) \pi
$$

Therefore $\operatorname{det}(D(\lambda))$ is zero whenever $\lambda$ is an eigenvalue, but it is also zero when $\lambda=4 p^{2}, p=1,2, \ldots$, which are not eigenvalues.

Although there does not appear to be any simple analytical means for removing these extraneous zeros, it should be noted that they are an artefact introduced by the elimination of the values $\left\{v_{\lambda}^{\prime}\left(x_{i}\right)\right\}_{i=0}^{N-1}$ and that they only arise non-trivially when $G_{i}\left(x_{i+1}, \lambda\right)$ and $G_{i+1}\left(x_{i+2}, \lambda\right)$ both vanish for some value of $\lambda$. This can only occur when $\lambda \geqslant \pi^{2} / h^{2}-\|s\|_{\infty}$. Thus, for most problems this difficulty either will not occur or will occur in a range for which the asymptotic expansion rather than the approximation method will be used.

\section{Approximation of differential equations not in normal form}

Although the technique of approximating the coefficients of the differential equation by piecewise constant functions provides a uniform approximation to the eigenvalues when the problem is in normal form, it can be shown that when this technique is applied to (1.1), Pruess' estimates (1.3) and (1.4) are sharp.

This suggests that when the problem is not in normal form, we should use the Liouville transformation to transform (1.1) into normal form. Thus if

$$
p, q, r \in C[a, b], \quad p r \neq 0 \text { and } p r \in C^{2}[a, b],
$$

then the Liouville transformation

$$
\begin{aligned}
& t=T^{-1} \int_{a}^{x}(r / p)^{\frac{1}{2}} d x, \quad T=\int_{a}^{b}(r / p)^{\frac{1}{2}} d x, \\
& u=w z, \quad w=(p r)^{-\frac{1}{4}}
\end{aligned}
$$

transforms (1.1) to

where

$$
-\ddot{z}+s z=\nu z, \quad t \in(0,1),
$$

and

$$
s(t)=T^{2}(g / r)(t)-(\ddot{w} / w)(t)+2(\dot{w} / w)^{2}(t)
$$

$$
\nu=T^{2} \lambda
$$


Similarly the boundary conditions become

where

$$
\begin{gathered}
\alpha^{*} z(0)+\beta \dot{z}(0)=0, \\
\gamma^{*} z(1)+\delta \dot{z}(1)=0,
\end{gathered}
$$

and

$$
\alpha^{*}=\alpha T(p / r)^{\frac{1}{2}}(0)+\beta(\dot{w} / w)(0)
$$

$$
\gamma^{*}=\gamma T(p / r)^{\frac{1}{2}}(1)+\delta(\dot{w} / w)(1) .
$$

We can then use the previously outlined method to obtain uniform approximations to the eigenvalues of this transformed problem and hence of the original problem. In some cases, however, it may not be possible to carry out this transformation explicitly. Thus, to obtain uniform approximations, it becomes necessary to introduce a further approximation.

From the form of the exactly transformed problem, it can be seen that the transformation parameter $T$, the function $s$ and the boundary condition coefficients $\alpha^{*}$ and $\gamma^{*}$ all need to be approximated. Let us therefore consider how we should perform these approximations:

From Theorem 2.1 it is clear that an $O(\varepsilon)$ perturbation of $s$ will produce, at most, an $O(\varepsilon)$ perturbation of the eigenvalues. Thus if $s(t)$ cannot be evaluated exactly, it is a relatively simple matter to approximate the desired quantities with sufficient accuracy so that the order of approximation of the eigenvalues is not affected.

The approximation of the parameter $T$, however, poses a more severe problem, since it is clear from (5.4) that a perturbation of $T$ will produce a perturbation in the eigenvalues which is proportional to the size of the eigenvalue. In practice, however, $T$ may be evaluated with sufficient accuracy so that any non-uniformity will not be apparent in the range of eigenvalues which are of interest.

The final problem is the effect of perturbations in $\alpha^{*}$ and $\gamma^{*}$. If these values cannot be found exactly, the conditions of Kato's theorem are no longer satisfied. We overcome this problem with

THEOREM 5.1. Let $\left\{\lambda_{k}(\sigma, \eta)\right\}_{k=0}^{\infty}$ be the ordered eigenvalues and $\left\{u_{k}(u, \sigma, \eta)\right\}_{k=0}^{\infty}$ be the corresponding normalized eigenfunctions of

where $s \in C[0,1]$. Then

$$
\begin{aligned}
-u^{\prime \prime}+s u & =\lambda u, \quad x \in(0,1), \\
\sigma u(0)-u^{\prime}(0) & =0, \\
\eta u(1)-u^{\prime}(1) & =0,
\end{aligned}
$$

$$
\left|\frac{\partial \lambda_{k}}{\partial \sigma}\right|,\left|\frac{\partial \lambda_{k}}{\partial \eta}\right| \leqslant M
$$

where $M$ is independent of $k$. 
Proof. It is clearly sufficient to show that the derivatives of $\lambda_{k}(\sigma, n)$ with respect to $\sigma$ and $\eta$ exist and are continuous. To do this we note that $\lambda_{k}(\sigma, \eta)$ is a continuous function of $\sigma$ and $\eta\left[6\right.$, p. 419] and also that $u_{k}(x, \sigma, \eta)$ depends continuously on $\sigma$ and $\eta[4$, p. 58$]$.

Let $u(x)=u_{k}(x, \sigma, \eta)$ and $\tilde{u}(x)=u_{k}(x, \tilde{\sigma}, \tilde{\eta})$, then

and

$$
-u^{\prime \prime}+s u=\lambda_{k}(\sigma, \eta) u
$$

Therefore

$$
-\bar{u}^{\prime \prime}+s \tilde{u}=\lambda_{k}(\tilde{\sigma}, \tilde{\eta}) \tilde{u} .
$$

$$
\left[\lambda_{k}(\sigma, \eta)-\lambda_{k}(\tilde{\sigma}, \tilde{\eta})\right] u \tilde{u}=-u^{\prime \prime} \tilde{u}+u \tilde{u}^{\prime \prime}
$$

and, on noting that

$$
\begin{aligned}
\int_{0}^{1}\left(-u^{\prime \prime} \tilde{u}+u \tilde{u}^{\prime \prime}\right) d x & =\left[-u^{\prime} \tilde{u}+u \tilde{u}^{\prime}\right]_{0}^{1} \\
& =u(0) \tilde{u}(0)(\sigma-\tilde{\sigma})-u(1) \tilde{u}(1)(\eta-\tilde{\eta}),
\end{aligned}
$$

we find on integrating (5.6),

$$
\left[\lambda_{k}(\sigma, \eta)-\lambda_{k}(\tilde{\sigma}, \tilde{\eta})\right] \int_{0}^{1} u \tilde{u} d x=u(0) \tilde{u}(0)(\sigma-\tilde{\sigma})-u(1) \tilde{u}(1)(\eta-\tilde{\eta}) .
$$

Now, since $u_{k}$ depends continuously on $\sigma$ and $\eta$, there is a $\delta=\delta(k)$ such that

$$
\int_{0}^{1} u \tilde{u} d x>0
$$

whenever $(\tilde{\sigma}, \tilde{\eta}) \in N_{\delta}(\sigma, \eta)=\left\{(x, y):(x-\sigma)^{2}+(y-\eta)^{2}<\delta^{2}\right\}$. Hence, from (5.7),

$$
\left[\lambda_{k}(\sigma, \eta)-\lambda_{k}(\tilde{\sigma}, \tilde{\eta})\right]=[u(0) \tilde{u}(0)(\sigma-\tilde{\sigma})-u(1) \tilde{u}(1)(\eta-\tilde{\eta})] / \int_{0}^{1} u \tilde{u} d x
$$

whenever $(\tilde{\sigma}, \tilde{\eta}) \in N_{\delta}(\sigma, \eta)$.

On taking the relevant limits and noting that

$$
\lim _{\tilde{\sigma} \rightarrow \sigma, \tilde{\eta}=\eta} \int_{0}^{1} u \tilde{u} d x=\lim _{\tilde{\sigma}=\sigma, \tilde{\eta} \rightarrow \eta} \int_{0}^{1} u \tilde{u} d x=\left\|u_{k}\right\|_{2}^{2}=1,
$$

we find that the derivatives of $\lambda_{k}$ with respect to $\sigma$ and $\eta$ exist, and

and

$$
\frac{\partial \lambda_{k}}{\partial \sigma}(\sigma, n)=u_{k}^{2}(0, \sigma, \eta)
$$

$$
\frac{\partial \lambda_{k}}{\partial \eta}(\sigma, \eta)=-u_{k}^{2}(1, \sigma, \eta)
$$

Thus the derivatives are continuous and bounded by $\left\|u_{k}^{2}\right\|_{\infty}$. The result then follows from the eigenfunction bounds in Lemma 3.1. 
Using these bounds in a standard mean value theorem shows that the perturbation of the eigenvalues is of the same order as the perturbations in $\alpha^{*}$ and $\gamma^{*}$. Thus, if $\alpha^{*}$ and $\gamma^{*}$ are approximated sufficiently well, the desired properties of the eigenvalue approximations will not be lost.

\section{Numerical examples}

To provide numerical confirmation of the preceding results, the method based on the three-term recurrence relation and outlined in Section 4 was implemented in a computer program. The determinant of the matrix was evaluated using Gaussian elimination with partial pivoting. The zeros were found using a modified secant method, the initial values for which were found by a fixed step search based on an initial prediction provided by an asymptotic expansion for the eigenvalues.

The first problem considered was

$$
\begin{aligned}
-\ddot{u}+t u & =\lambda u, \quad t \in(0,1), \\
u(0) & =0=\dot{u}(1) .
\end{aligned}
$$

The approximating problem was chosen to be that given by approximating $s$ using a midpoint approximation rule on a uniform partition with $N$ intervals.

The first forty eigenvalues of this approximating problem for $N=16$ were found, and an estimate of the error was obtained by comparing these results with

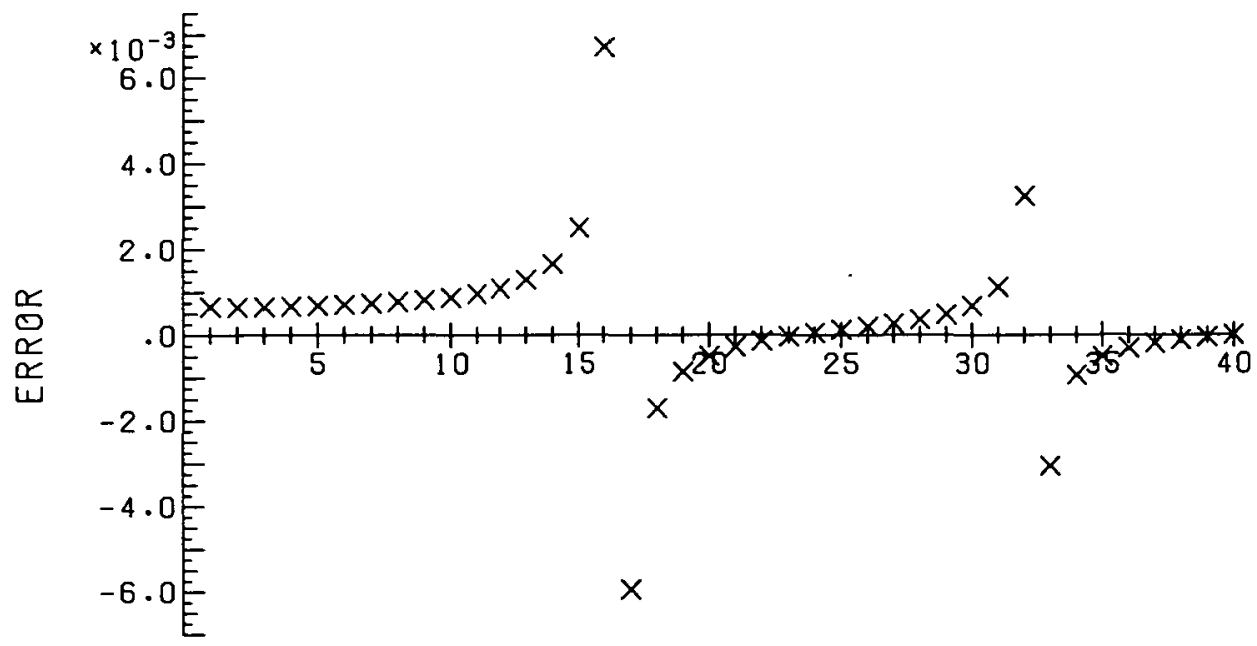

\section{EIGENVALUE NUMBER}

Fig. 1. Eigenvalue error for problem I. 
those obtained for $N=256$. The errors thus obtained are plotted in Fig. 1, and it should be noted that they appear to be in close agreement with the bound given in Theorem 3.1.

At this stage it is worthwhile pointing out that for this example, the $O(h)$ peaks are in apparent disagreement with the asymptotic formula equation (42) of [14] which predicts that the error in this example should decrease like $O\left(k^{-2}\right)$. However, the observation that this asymptotic formula requires $s$ and $\tilde{s}$ to be continuously differentiable resolves this contradiction since this condition is clearly not satisfied by piecewise constant approximations.

From the above figure and comments it is clear this method will not directly yield the desired uniform approximations and so the strategy of switching to the asymptotic expansion (3.5) will have to be used. The error in the approximations obtained in this manner for the above example (again with $N=16$ ) is presented in Fig. 2 and clearly shows that this is a viable strategy for obtaining uniform approximations.

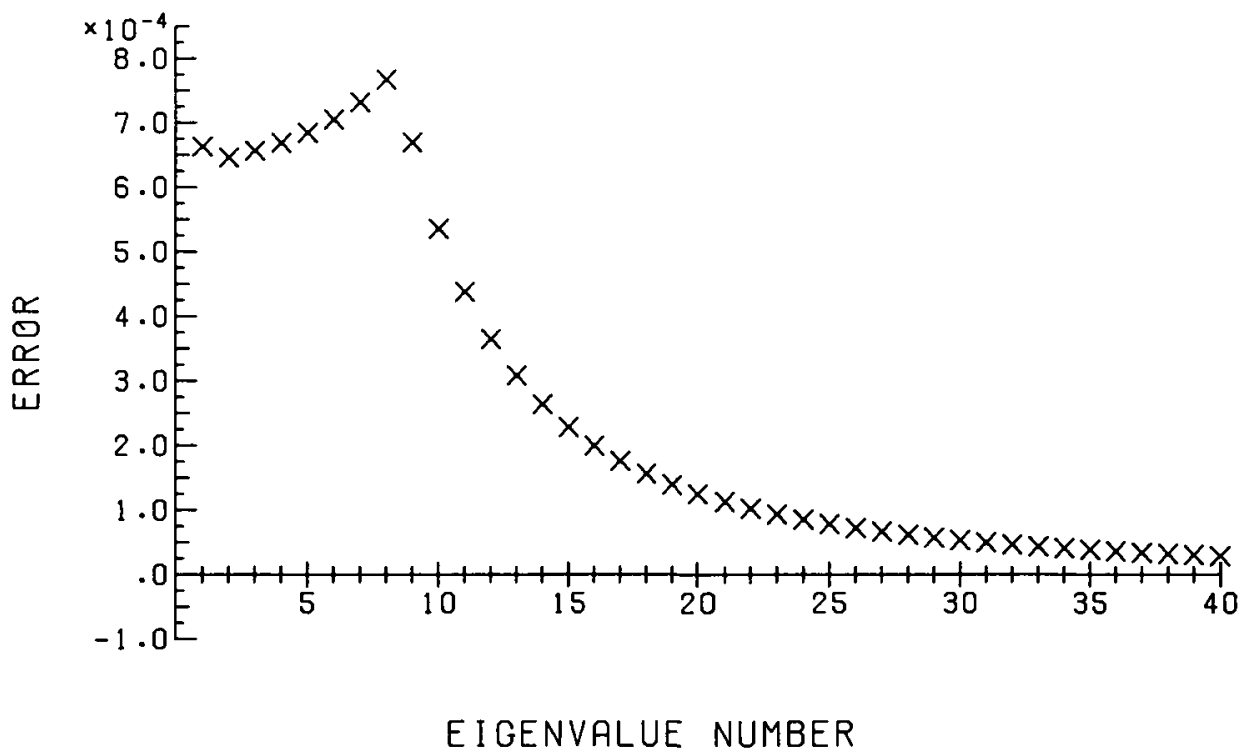

Fig. 2. Eigenvalue error using approximation of differential equation and asymptotic expansion for problem 1 .

The next problem considered was

$$
\begin{aligned}
-\left(x u^{\prime}\right)^{\prime}+\frac{\ln x}{x} u & =\frac{\lambda}{x} u, \quad x \in(1, e), \\
u(1) & =0=u^{\prime}(e)
\end{aligned}
$$


which was obtained from the first problem via the transformation $x=e^{t}$. This was solved using the approximate transformation technique outlined in the previous section.

The method used was firstly to partition the $x$-interval, and then use Simpson's rule to approximate the transformation (5.1). This yields a partition of the $t$ interval and an approximation of the parameter $T$, which in this case was evaluated to an accuracy of $1.0 \times 10^{-6}$. The next step was to approximate the boundary conditions. This was achieved by using finite differences to approximate the required derivatives, the mesh being refined until a specified accuracy (in this case $1.0 \times 10^{-4}$ ) was achieved. The final step was to approximate $s$ by the use of centred finite difference approximations on the previously obtained partition of the $t$ interval. The method outlined previously for problems in normal form was then used to obtain the eigenvalues of this approximating problem.

This procedure was carried out for two partitions of the $x$-interval. The first was a uniform partition, and the results obtained, which are plotted in Fig. 3, show that accurate approximations are obtained, although there appears to be no clear structure in the error.

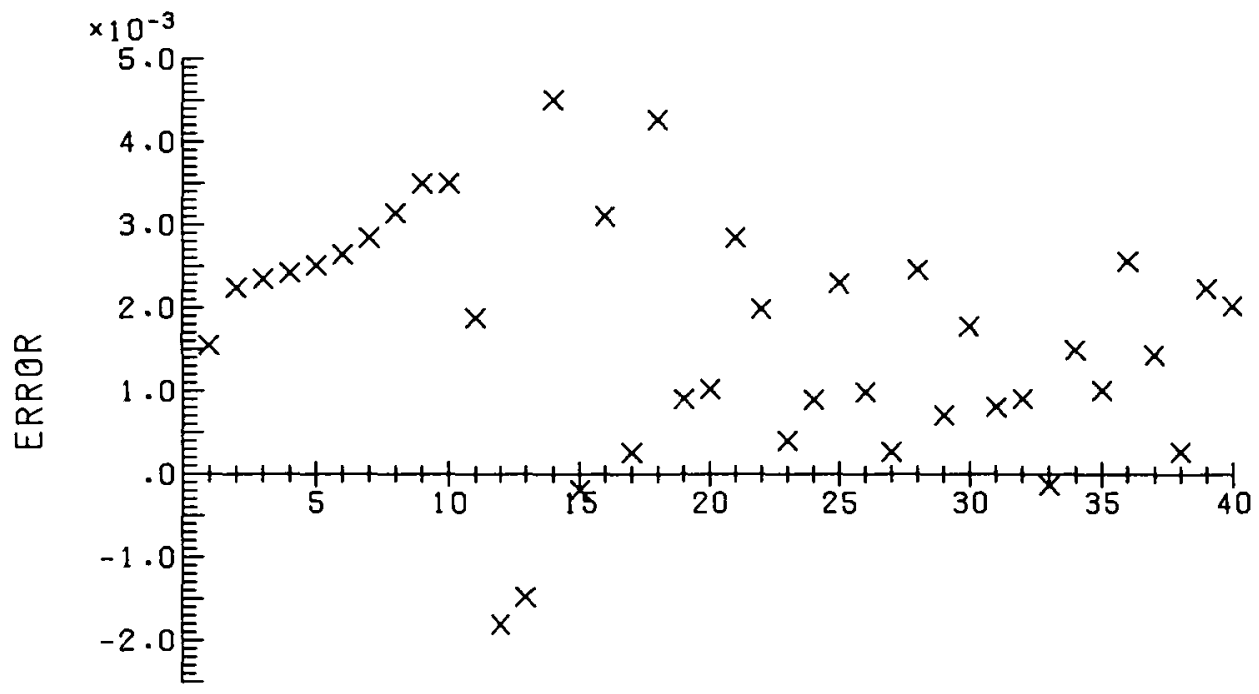

EIGENVALUE NUMBER

Fig. 3. Eigenvalue error for problem II.

The second partition used was one which, when exactly transformed, yielded a uniform partition of the $t$-interval. The results obtained for this approximation did not differ significantly from those obtained from the exactly transformed problem, that is, the first test problem. 


\section{References}

[1] R. S. Anderssen and J. R. Cleary, "Asymptotic structure in torsional free oscillations of the earth-I. Overtone structure", Geophys. J.R. Astr. Soc. 39 (1974), 241-268.

[2] G. Birkhoff, C. de Boor, B. Swartz and B. Wendroff, "Rayleigh-Ritz approximation by piecewise cubic polynomials", SIAM J. Numer. Anal. 3 (1966), 188-203.

[3] J. Canosa and R. G. de Oliveira, "A new method for the solution of the Schrödinger equation", J. Comp. Phys. 5 (1970), 188-207.

[4] E. Coddington and N. Levinson, Theory of ordinary differential equations (McGraw-Hill, New York, 1955).

[5] S. D. Conte and C. de Boor, Elementary numerical analysis: an algorithmic approach (McGraw-Hill, New York, 1972).

[6] R. Courant and D. Hilbert, Methods of mathematical physics, Vol. I (Interscience, New York, 1953).

[7] R. Gordon, "New method for constructing wave functions for bound states and scattering", J. Chem. Phys. 51 (1969), 14-25.

[8] R. T. Gregory and D. T. Karney, A collection of matrices for testing computational algorithms (Wiley-Interscience, New York, 1969).

[9] N. A. Haskell, "The dispersion of surface waves on multilayered media", Bull. Seismol. Soc. Amer. 43 (1953), 17-34.

[10] L. Gr. Ixaru, "The error analysis of the algebraic method for solving the Schrödinger equation", J. Comp. Phys. 9 (1972), 159-163.

[11] T. Kato, Perturbation theory for linear operators (Springer-Verlag, Berlin, 1966).

[12] M. R. Osborne, "Numerical procedures for the eigenvalue problems of second order ordinary differential equations when a wide range of eigenvalues are required". In Actas del seminario sobre métodos numéricos modernos, Vol. II (Caracas, 1974).

[13] M. R. Osborne and S. Michaelson, "The numerical solution of eigenvalue problems in which the eigenvalue parameter appears non-linearly, with an application to differential equations", Computer J. 7 (1964), 66-71.

[14] S. Pruess, "Estimating the eigenvalues of Sturm-Liouville problems by approximating the differential equation", SIAM J. Numer. Anal. 10 (1973), 55-68.

[15] S. Pruess, "High order approximations to Sturm-Liouville eigenvalues", Numer. Math. 24 (1975), 241-247.

[16] M. H. Schultz, Spline analysis (Prentice Hall, Englewood Cliffs, N.J., 1973).

[17] M. H. Stone, Linear transformations in Hilbert space (American Mathematical Society, New York, 1932).

[18] C. Wang, J. F. Gettrust and J. R. Cleary, "Asymptotic overtone structure in eigenfrequencies of torsional normal modes of the earth: a model study", Geophys. J.R. Astr. Soc. 50 (1977), 289-302.

\section{Computing Research Group}

Australian National University

P.O. Box 4, Canberra, A.C.T. 2600

Australia

and

C.S.I.R.O. Division of Mathematics and Statistics

Yarralumla, A.C.T. 2600

Australia 